American Journal of Economics and Business Administration 4 (1): 72-83, 2012

ISSN 1945-5488

(C) 2012 Science Publications

\title{
Public Funding and Affordability of Substance Abuse Treatment Services
}

\author{
${ }^{1}$ Roberto A. Trevino and ${ }^{2}$ Alan J. Richard \\ ${ }^{1}$ Department of FACIS, College of Business, \\ University of Houston-Downtown, \\ One Main St, Houston, TX 77002-1001, USA \\ ${ }^{2}$ RTH Research Group, \\ LLC 7322 SW Freeway, Suite 1100, Houston, TX 77074, USA
}

\begin{abstract}
Problem statement: Substance abuse continues to cause significant personal, social and economic costs to the US economy. In order to decrease these costs, the goal of the US public health policy has been to increase access to drug treatment services by increasing their affordability. Government policy makers try to achieve such a goal by providing direct public funding to private non-profit Drug Treatment Providers (DTPs) via grants and earmarks. It is argued that public funding will allow DTPs to offer lower prices for treatment services thus making them more affordable to people who need them. Although many DTPs depend heavily on government grants, there is little research examining the effect that public funding has on the affordability of treatment services. This study examines whether public funding increases the affordability of treatment services or whether instead there is a "flypaper effect" of such aid. The flypaper effect occurs when an exogenous aid sticks where it hits and thus fails to have the intended effect. Approach: This study examines the degree to which the price of treatment services is a function of public funding, net of DTP size, local socioeconomic conditions and state policy, using Hierarchical Linear Modeling (HLM) to adjust for endogeneity resulting from clustering of cases. Data from a 2009 national cross-sectional data set of DTPs are used for analysis. Results: The results show that when the data are analyzed using linear regression models, DTPs that receive public funding are statistically more likely to offer lower prices for their services. However, when Hierarchical Linear Models (HLM) are used, this effect diminishes and even disappears in some cases. Conclusion/Recommendations: The results of this study suggest that in order to maximize the benefits of the limited public funds available and make drug treatment services more affordable, public funds need to have stronger regulatory oversight as to ensure that these limited funds increase treatment affordability as intended.
\end{abstract}

Key words: Substance abuse, Drug Treatment Providers (DTPs), flypaper effect, drug treatment services, Hierarchical Linear Models (HLM), treatment affordability

\section{INTRODUCTION}

It is estimated that substance abuse costs over $\$ 600$ billion a year to the US economy (Harwood and Bouchery, 2004; CDCP, 2007; Rehm et al., 2009). The National Household Survey on Drug Use and Health estimates that almost $10 \%$ or an estimated 22.6 million Americans (12 years old or older) used some type of illicit drug in 2010. The same data source indicates that illicit drug use has increased in the last few years and that the prevalence of illicit drug use centers around large metropolitan areas primarily in the West and Northeast of the US.

At the same time, however, more than 25 years of research have shown that substance abuse treatment is effective (McLellan et al., 1982; O'Brien and Jaffe, 1992). Therefore, the goal of US public health policy has been to increase access to substance abuse treatment services by increasing its affordability. Increased affordability is achieved indirectly by providing for the financing of treatment services, via Medicaid and Medicare and directly by providing direct funding allocations, via grants and earmarks, for non-profit private Drug Treatment Providers (DTPs). In 2010, the US Substance Abuse and Mental Health Services Administration (SAMHSA) allocated about $\$ 2.1$ billion to States for substance abuse treatment services (1.6 billion in block grants and 0.5 billion in discretionary grants). (This amount does not include the monies allocated for

Corresponding Author: Roberto A. Trevino, Department of FACIS, College of Business, University of Houston-Downtown, One Main St, Houston, TX 77002-1001, USA Tel: 713-222-5379 
substance abuse treatment services via Medicaid, Medicare, Health Resources and Services Administration and Criminal Justice System allocations.) Despite these apparently high figures (as percentage of all government expenditures) and that treatment has been found to be cost effective, drug treatment continues to be seriously underfunded in the US (Meara and Frank, 2005).

Behavioral health services researchers have examined how internal DTP factors help explain both DTP processes and outcomes. For example, Olmstead and Sindelar $(2004 ;$ 2005) has examined how managed care affected the supply of treatment services. Montoya (2006) examined how for-profit status affects the supply of auxiliary drug treatment services. Mojtabai (2004) examined how DTP characteristics affected the supply of substance abuse for the dually diagnosed; Campbell and Alexander (2006); Campbell et al. (2009) and Nahra et al. (2009) examined the effect of ownership on access to treatment; and Grella and Greenwell (2004) examined how treatment services for women vary over time across treatment providers. Brown et al. (2011) examined how child care affected treatment services for women and Cochran et al. (2007) examined the availability of treatment services for gay and bisexual clients. The current study adds to this important area of research by examining whether direct public funding made to DTPs increases affordability of substance abuse treatment services.

Although Federal Block Grants are allocated to States based on formula, many other Federal substance abuse treatment public funds are allocated to States and to DTPs on a competitive basis. (Examples of funds directly allocated to DTPs by SAMHSA include the Target Capacity Expansion and the Community Reinforcement Approach Programs. Grants directed to States include the Brief Intervention Programs.) This study will focus on funds directly allocated to DTPs. In order to be awarded these funds, the applicant (DTPs) must show that there is a need for additional funding to either increase the capacity of treatment or the affordability of treatment for the targeted population in their chosen target area. In addition, when requesting these funds, DTPs must outline specific objectives that the grant funds will accomplish. However, even though the grant funds are allocated for a specific use, the recipient (DTPs) can decrease the amount that currently allocates to another targeted category and replaces them with the grant funds and transfer the released funds to another category chosen by the DTP. The chosen category may or may not be associated (directly or indirectly) with the category outlined in the grant. That is, aid is fungible. Thus the grant funds may not have an impact on the specified grant objective.

The process by which aid becomes fungible and thus fails to achieve the intended effect has received the research interest of public finance economists for many years. The public finance theory argues that when a higher level of government, such as the Federal government, allocates funds to a lower system of government, such as a State/City or to a non-for-profit agencies, such as a DTP, the exogenous funds should have the same effect as an equal corresponding increase in local tax revenue. But research has shown that when higher government agencies allocate funds to lower level agencies, there is higher public spending than an equivalent increase from private-citizen's income. This process has been referred to as the "flypaper effect". That is, money sticks where it hits. Public finance researchers have examined the existence of the flypaper effect in general government expenditures made (Bae and Feiock, 2004; Schwallie, 1989), highway allocations (Nesbit and Kreft, 2009; Knight, 2002), public assistance programs (Moffitt, 1984), foreign aid (Walle and $\mathrm{Mu}, 2007$; Tamura, 2005) and on intrahousehold allocations (Jacoby, 2002; Islam and Hoddinott, 2009).

However, public choice theory argues that since the median voter theorem suggests that government agencies' expenditures act as agents of this median voter, any higher increase in expenditures resulting from exogenous aid-is an anomaly. Researchers do not yet agree on what causes this effect or whether there is an anomaly to the median voter theory. Researchers have explained this anomaly based on specification error, measurement error and on the complete denial of such anomaly (Hines and Thaler, 1995; Roemer and Silvestre, 2002; Strumpf, 1996; Karnik and Lalvani, 2008; Turnbull, 1998). This study will not contribute to this important debate in public choice theory, but instead will focus on whether there is a flypaper effect on the allocation of public funding for substance abuse treatment, a priori of any potential flypaper effect anomaly.

Specific research on the flypaper effect of public funding allocations for substance abuse treatment is limited. Cowell et al. (2003) and Heinrich and Fournier (2004) are the only researchers to have examined this issue. Although they do not empirically estimate the effect of federal substance abuse funding allocations on the State expenditures, they provide a meta-analysis of the issues sounding the flypaper effect and its relation to the Federal substance abuse treatment block grant. Their study concludes that although there is no clear evidence of the existence of a flypaper effect, there is strong evidence that the substance abuse treatment 
block grant does not reduce State expenditures on substance abuse treatment. This study builds on this work by specifically examining whether public funds allocated to DTPs by a higher funding agency have an effect on the affordability of substance abuse treatment services or if instead there is a flypaper effect of these exogenous public funding allocations.

\section{MATERIALS AND METHODS}

Data from the National Survey of Substance Abuse Treatment Services (NSSATS) for 2009 were used in the analyses. The NSSATS contains information about the characteristics of the DTPs such as ownership type (public, private non-profit, private for-profit), types of payments accepted (fee/pay scale, no charge/free) and whether the DTP receives public funding from federal, state or local governments. The NSSATS does not provide details on the amount of this aid, or what percentage of the DTP's total budget is based on public funding allocations. The NSSATS also provides information about the characteristics of DTPs such as whether substance abuse treatment is their primary focus, modality of treatment (residential or outpatient), number of clients, clients with alcohol and drug dependency disorders, external accreditations, type of ancillary services provided (employment assistance, transportation, housing assistance among others) and type of specialized treatment services provided (gay/bisexual clients, women, criminal justice, among others).

The goal of this study is to examine whether DTPs that receive public funding allocations are more likely to provide lower payment options to their clients than DTPs that do not receive public funding. In particular, the study examines the extent to which public funding increases affordability of substance abuse treatment services. Affordability is measured by whether the DTP offers treatment based on the following three types of price options: fee/pay Scale (SCALE), no charge/Free (FREE) and/or Both (BOTH). To examine the effect of public funding on the affordability of treatment services, the study first estimates the following linear regression model:

$$
\begin{aligned}
& \text { (1) } Y_{i}=\left\{\begin{array}{l}
\text { SCALE } \\
\text { FREE } \\
\text { BOTH }
\end{array}\right\}=\beta_{0}+\beta_{1}(\text { PUBFUND })+\beta_{2}(\text { DTPSIZE }) \\
& +\beta_{3}(\text { MSASES })+\beta_{4}(\text { STGOV })+\varepsilon_{\mathrm{i}}
\end{aligned}
$$

where, subscript $\mathrm{i}$ measures the DTP and $\mathrm{Y}$ is a dependent dichotomous variable $(1=$ Yes, $0=$ No $)$ representing the DTP's price behavior as measured by whether the DTP offers treatment services based on fee/pay Scale (SCALE), free of charge (FREE) or Both (BOTH). Pubfund is a dummy variable $(1=$ Yes, $0=$ No) indicating whether the DTP receives public funding. Since only private non-profits DTPs are generally granted public funding, the analyses are based only on private non for-profit DTPs. DTPSIZE represents the size of the DTP as measured by the number of clients $(1=$ less than 25 clients, $5=300$ clients or more).

The NSSATS also provides data on DTP characteristics such as whether the DTP is a substance abuse focused provider, whether DTP offers residential substance abuse treatment -in addition to outpatient services, percentage of clients diagnosed with both alcohol and drug dependence and type of ancillary and specialized services provided. Although these variables were included in the original estimation, the model failed to converge and thus they were not included in the final regression analyses. In addition, these variables were highly correlated with other independent variables. MSASES measures the normalized Socio Economic Status of the MSA where DTPs are located. MSASES is normalized and weighted by per-capita income, percentage of college graduates and unemployment rate (Daly et al., 2002). STGOV is a proxy to measure States' support for drug treatment services and it is measured by whether the DTP is located in a State with a Republican Governor (1) or a Democratic Governor (0). Finally, $\varepsilon_{\mathrm{i}}$ denotes a random error term.

The next step in the analyses was to test for the appropriate empirical specification of equation 1 given that the data are clustered. In other words, DTPs are nested within MSAs, which are nested within States. We used Hierarchical Linear Models (HLM) rather than linear regression models to overcome the issues associated with clustered/nested level data (Raudenbush and Bryk, 2002). Researchers often face similar data issues. For instance, educational researches face data clustering when examining academic achievement among students nested within classes, which are nested within schools. Organizational psychologists face data clustering when examining employee job satisfaction when employees are nested within departments and the latter are nested within firms. Family psychologists face it when examining children's behavioral problems, when children are nested within families within communities (Raudenbush and Bryk, 2002).

Therefore, in this study and in order to account for nested/clustered data, DTPs were clustered in Level 1, MSAs were clustered in Level 2 and States were clustered in Level 3. For notation purposes, we will denote SCALE, FREE, BOTH (the dependent variables) by Y. A total of five HLM were estimated and they are outlined in Table 1. The first HLM 
Model estimated (HLM1) is an unconditional model and it is specified as follows.

\section{HLM1 Level 1:}

Prob $=\left(\mathrm{Y}_{\mathrm{ijk}}=1 \mid \pi_{\mathrm{jk}}\right)=\phi_{\mathrm{ijk}}$
$\log =\left[\phi_{\mathrm{ijk}} /\left(1-\phi_{\mathrm{ijk}}\right)\right]=\eta_{\mathrm{ijk}}$
$\eta_{\mathrm{ijk}}=\pi_{0 \mathrm{jk}}$

where the indices $\mathrm{i}, \mathrm{j}, \mathrm{k}$ denote DTPs, MSAs and States, respectively. Thus, for Level $1, \mathrm{Y}_{\mathrm{ijk}}$ measures whether $\mathrm{DTP}_{\mathrm{i}}$ in $\mathrm{MSA}_{\mathrm{j}}$, in State $_{\mathrm{k}}$ provides treatment services based on SCALE, FREE or BOTH. $\mathrm{Y}_{\mathrm{ijk}}$ is a binary outcome (Yes $=1$, No $=0$ ) and is assumed to have a
Bernoulli distribution and $\varnothing_{\mathrm{ijk}}$ is the probability of success (yes) on $\mathrm{m}_{\mathrm{ijk}}$ trails. That is, $\mathrm{Y}_{\mathrm{ijk}} \mid \varphi_{\mathrm{ijk}} \sim \mathrm{B}\left(\mathrm{m}_{\mathrm{ijk}}, \varphi_{\mathrm{ijk}}\right)$ and its expected value and variance are given by: $\mathrm{E}\left(\mathrm{Y}_{\mathrm{ijk}} \mid \varphi_{\mathrm{ijk}}\right)=\mathrm{m}_{\mathrm{ijk}}, \varphi_{\mathrm{ijk}}$ and $\operatorname{Var}\left(\mathrm{Y}_{\mathrm{ijk}} \mid \varphi_{\mathrm{ijk}}\right)=\mathrm{m}_{\mathrm{ijk}}, \varphi_{\mathrm{ijk}}\left(1-\varphi_{\mathrm{ijk}}\right)$, respectively. Lastly, $\quad$ since $_{\eta_{i j k}}=\log \left(\frac{\varphi_{i j k}}{1-\varphi_{i j k}}\right)$, the predicted log-odds of $\eta_{\mathrm{ijk}}=\exp \left(\eta_{\mathrm{ijk}}\right)$, yields a predicted probability between 0 and 1 and it can be estimated by $\phi_{\mathrm{ijk}}=\left[\frac{1}{1+\exp \left(-\eta_{\mathrm{ijk}}\right)}\right]$. At level 2, HLM1 is given by.

Table 1: HLM models estimated

Dependent Variables: ATS = PAY/FEE SCALE (Model A), FREE SERVICES (Model B), BOTH (Model C)

HLM1: unconditional model $\quad$ HLM2: Conditional model, w/o controlling variables on LV1

$\operatorname{Prob}\left(\mathrm{ATS}_{\mathrm{ijk}}=1 \mid \pi_{\mathrm{jk}}\right)=\phi_{\mathrm{ijk}}$

$\log \left[\phi_{\mathrm{ijk}} /\left(1-\phi_{\mathrm{ijk}}\right)\right]=\eta_{\mathrm{ijk}}$

$\eta_{\mathrm{ijk}}=\pi_{0 \mathrm{jk}}$

Level-2 Model

$\pi_{0 \mathrm{jk}}=\beta_{00 \mathrm{k}}+\mathrm{r}_{0 \mathrm{jk}}$

Level-3 Model

$\beta_{00 k}=\gamma_{000}+u_{00 k}$

Level-1 Model

$\operatorname{Prob}\left(\operatorname{ATS}_{\mathrm{ijk}}=1 \mid \pi_{\mathrm{jk}}\right)=\phi_{\mathrm{ijk}}$

$\log \left[\phi_{\mathrm{ijk}} /\left(1-\phi_{\mathrm{ijk}}\right)\right]=\eta_{\mathrm{ijk}}$

$\eta_{\mathrm{ijk}}=\pi_{0 \mathrm{jk}}+\pi_{\mathrm{jjk}} *\left(\mathrm{PUBFUND}_{\mathrm{ijk}}\right)$

Level-2 Model

$\pi_{0 \mathrm{jk}}=\beta_{00 \mathrm{k}}+\mathrm{r}_{0 \mathrm{jk}}$

$\pi_{1 \mathrm{jk}}=\beta_{10 \mathrm{k}}+\mathrm{r}_{1 \mathrm{jk}}$

Level-3 Model

$\beta_{00 k}=\gamma_{000}+u_{00 k}$

$\beta_{10 \mathrm{k}}=\gamma_{100}+\mathrm{u}_{10 \mathrm{k}}$

HLM3: Conditional Model, w/ controlling variables in

HLM4: Random Slope and intercept model w/Level 2 controls

LV1 and no controls in Level 2 and 3

Level-1 Model

$\operatorname{Prob}\left(\mathrm{ATS}_{\mathrm{iijk}}=1 \mid \pi_{\mathrm{jk}}\right)=\phi_{\mathrm{ijk}}$

$\log \left[\phi_{\mathrm{ijk}} /\left(1-\phi_{\mathrm{ijk}}\right)\right]=\eta_{\mathrm{ijk}}$

$\eta_{\mathrm{ijk}}=\pi_{0 \mathrm{jk}}+\pi_{1 \mathrm{j} \mathrm{k}} *\left(\right.$ PUBFUND $\left._{\mathrm{ijk}}\right)+\pi_{2 \mathrm{j} \mathrm{k}} *\left(\right.$ DTPSIZE $\left._{\mathrm{ijk}}\right)$

Level-2 Model

$\pi_{0 \mathrm{ik}}=\beta_{00 \mathrm{k}}+\mathrm{r}_{0 \mathrm{jk}}$

$\pi_{1 \mathrm{jk}}=\beta_{10 \mathrm{k}}+\mathrm{r}_{1 \mathrm{j} \mathrm{k}}$

$\pi_{2 \mathrm{jk}}=\beta_{20 \mathrm{k}}+\mathrm{r}_{2 \mathrm{jk}}$

Level-3 Model

$\beta_{00 \mathrm{k}}=\gamma_{000}+\mathrm{u}_{00 \mathrm{k}}$

$\beta_{10 \mathrm{k}}=\gamma_{100}+\mathrm{u}_{10 \mathrm{k}}$

$\beta_{20 \mathrm{k}}=\gamma_{200}+\mathrm{u}_{20 \mathrm{k}}$

Level-1 Model

$\operatorname{Prob}\left(\operatorname{ATS}_{\mathrm{ijk}}=1 \mid \pi_{\mathrm{jk}}\right)=\phi_{\mathrm{ijk}}$

$\log \left[\phi_{\mathrm{ijk}} /\left(1-\phi_{\mathrm{ijk}}\right)\right]=\eta_{\mathrm{ijk}}$

$\eta_{\mathrm{ijk}}=\pi_{0 \mathrm{jk}}+\pi_{1 \mathrm{jj}} *\left(\right.$ PUBFUND $\left._{\mathrm{ijk}}\right)+\pi_{2 \mathrm{j} \mathrm{k}} *\left(\right.$ DTPSIZE $\left._{\mathrm{ijk}}\right)$

Level-2 Model

$\pi_{0 \mathrm{ik}}=\beta_{00 \mathrm{k}}+\beta_{01 \mathrm{k}} *\left(\right.$ MSASES $\left._{\mathrm{ik}}\right)+\mathrm{r}_{0 \mathrm{ik}}$

$\pi_{1 \mathrm{jk}}=\beta_{10 \mathrm{k}}+\beta_{11 \mathrm{k}} *\left(\right.$ MSASES $\left._{\mathrm{jk}}\right)+\mathrm{r}_{1 \mathrm{jk}}$

$\pi_{2 \mathrm{jk}}=\beta_{20 \mathrm{k}}+\beta_{21 \mathrm{k}} *\left(\right.$ MSASES $\left._{\mathrm{jk}}\right)+\mathrm{r}_{2 \mathrm{jk}}$

Level-3 Model

$\beta_{00 \mathrm{k}}=\gamma_{000}+\mathrm{u}_{00 \mathrm{k}}$

$\beta_{01 \mathrm{k}}=\gamma_{010}+\mathrm{u}_{01 \mathrm{k}}$

$\beta_{10 \mathrm{k}}=\gamma_{100}+\mathrm{u}_{10 \mathrm{k}}$

$\beta_{11 \mathrm{k}}=\gamma_{110}+\mathrm{u}_{11 \mathrm{k}}$

$\beta_{20 \mathrm{k}}=\gamma_{200}+\mathrm{u}_{20 \mathrm{k}}$

$\beta_{21 \mathrm{k}}=\gamma_{210}+\mathrm{u}_{21 \mathrm{k}}$

HLM5: Random intercept slope model w/level 2 and

level 3 controls

Level-1 Model

$\operatorname{Prob}\left(\mathrm{ATS}_{\mathrm{ijk}}=1 \mid \pi_{\mathrm{jk}}\right)=\phi_{\mathrm{ijk}}$

$\log \left[\phi_{\mathrm{ijk}} /\left(1-\phi_{\mathrm{ijk}}\right)\right]=\eta_{\mathrm{ijk}}$

$\eta_{\mathrm{ijk}}=\pi_{0 \mathrm{jk}}+\pi_{1 \mathrm{jk}} *\left(\right.$ PUBFUND $\left._{\mathrm{ijk}}\right)+\pi_{2 \mathrm{jk}} *\left(\right.$ DTPSIZE $\left._{\mathrm{ijk}}\right)$

Level-2 Model

$\pi_{0 \mathrm{ik}}=\beta_{00 \mathrm{k}}+\beta_{01 \mathrm{k}} *\left(\right.$ MSASES $\left._{\mathrm{ik}}\right)+\mathrm{r}_{0 \mathrm{ik}}$

$\pi_{1 \mathrm{jk}}=\beta_{10 \mathrm{k}}+\beta_{11 \mathrm{k}} *\left(\right.$ MSASES $\left._{\mathrm{jk}}\right)+\mathrm{r}_{1 \mathrm{j} \mathrm{k}}$

$\pi_{2 \mathrm{jk}}=\beta_{20 \mathrm{k}}+\beta_{21 \mathrm{k}} *\left(\right.$ MSASES $\left._{\mathrm{jk}}\right)+\mathrm{r}_{2 \mathrm{jk}}$

Level-3 Model

$\beta_{00 \mathrm{k}}=\gamma_{000}+\gamma_{001}\left(\mathrm{STGOV}_{\mathrm{k}}\right)+\mathrm{u}_{00 \mathrm{k}}$

$\beta_{01 \mathrm{k}}=\gamma_{010}+\gamma_{011}\left(\mathrm{STGOV}_{\mathrm{k}}\right)+\mathrm{u}_{01 \mathrm{k}}$

$\beta_{10 \mathrm{k}}=\gamma_{100}+\gamma_{101}\left(\mathrm{STGOV}_{\mathrm{k}}\right)+\mathrm{u}_{10 \mathrm{k}}$

$\beta_{11 \mathrm{k}}=\gamma_{110}+\gamma_{111}\left(\right.$ STGOV $\left._{\mathrm{k}}\right)+\mathrm{u}_{11 \mathrm{k}}$

$\beta_{20 \mathrm{k}}=\gamma_{200}+\gamma_{201}\left(\mathrm{STGOV}_{\mathrm{k}}\right)+\mathrm{u}_{20 \mathrm{k}}$

$\underline{\beta_{21 \mathrm{k}}=\gamma_{210}+\gamma_{211}\left(\mathrm{STGOV}_{\mathrm{k}}\right)+\mathrm{u}_{21 \mathrm{k}}}$ 


\section{HLM1 Level-2:}

$\pi_{0 \mathrm{jk}}=\beta_{00 \mathrm{k}}+\mathrm{r}_{0 \mathrm{jk}}$

where, $\beta_{00 \mathrm{k}}$ is the mean of $\mathrm{Y}_{\mathrm{k}}$ in State $\mathrm{k}$ and $\mathrm{r}_{0 \mathrm{jk}}$ is a random "MSA effect" which captures the deviation of $\mathrm{MSA}_{\mathrm{jk}}$ 's mean from the State ${ }_{\mathrm{k}}$ 's mean. And at level 3, HLM1 is as follows:

\section{HLM1 Level-3:}

$\beta_{00 \mathrm{k}}=\gamma_{000}+\mathrm{u}_{00}$

where, $\beta_{00 \mathrm{k}}$ is modeled as varying, randomly, around a grand mean, $\gamma_{000}$ and $u_{00 k}$ is random effect which captures the "State effect" or the deviation of State k's mean from the grand mean $\gamma_{000}$.

Next, the study estimates for conditional HLM models. Again, the dependent variables (SCALE, FREE, BOTH) are denoted by Y. The first conditional model (HLM2) levels are given as follows:

\section{HLM2 Level-1:}

Prob $=\left(\mathrm{Y}_{\mathrm{ijk}}=1 \mid \pi_{\mathrm{jk}}\right)=\phi_{\mathrm{ijk}}$

$\log =\left[\phi_{\mathrm{ijk}} /\left(1-\phi_{\mathrm{ijk}}\right)\right]=\eta_{\mathrm{ijk}}$

$\eta_{\mathrm{ijk}}=\pi_{0 \mathrm{jk}}+\pi_{1 \mathrm{jk}} *\left(\right.$ PUBFUND $\left._{\mathrm{ijk}}\right)$

where, PUBFUND denotes whether DTP $_{i}$ in $\mathrm{MSA}_{\mathrm{j}}$, in State $_{\mathrm{k}}$ received public funding. Level 2 and level 3 for HLM2 remain the same as in HLM1.

For HLM3, DTPSIZE is added as a controlling variable at level 1. Level 2 and level 3 remain the same as in HLM2. For HLM4, referred as random slope and intercept models, level 1 and level 3 are the same as specified in HLM3, but level 2 includes MSASES as a controlling variable. Finally, under HLM5, level 1 and level 2 remain as in HLM4, but level 3 includes STGOV as a controlling variable. Again, the specific HLM models estimated are outlined in Table 1. For each of the HLM models aforementioned, substituting Level 3 into Level 2 and then into Level 1 provides the mixed or fixed and random effects model.

\section{RESULTS}

All the six aforementioned models were estimated using data on 6,062 private non-profit DTPs in 359 MSAs with populations greater than 100,000 people in 50 States. Table 2 shows the characteristics of the DTPs, MSAs and State variables used in the analyses. First, as regards treatment payment options offered by the DTPs (dependent variable), about $60 \%$ of DTPs offered treatment services based on either a pay/fee scale or at no charge and slightly less than $50 \%$ of them offered both payment options. In regards to public funding (PUBFUND), almost 3 in 4 of them received public funding from Federal, State or Local governments. Again, the data did not provide details on the amount of such aid or the percentage that such funding represented out of the DTP's total budget.

With regards to other DTP characteristics, the majority of the DTPs $(64 \%)$ specialized in providing substance abuse services and more than half of them $(56 \%)$ treated clients diagnosed with both drug and alcohol dependence. Only about $25 \%$ of them provided specialized treatment services, but about $55 \%$ of them provided auxiliary treatment services. Around 1/3 of them provided treatment in a residential setting in addition to an outpatient setting and the vast majority of DTPs were small to medium size (2.56) with an average number of clients of 112. Level 2 and level 3 descriptive statistics are also provided in Table 2. Most DTPs were located in MSAs with a medium-level SES score and slightly less than half of the DTPs were located in States that had a Republican Governor.

Table 3A shows treatment payment options offered by DTPs based on public funding received. The data show that DTPs that received public funding were significantly $(\mathrm{p}<0.05)$ more likely to offer treatment services based on a fee/pay scale than DTPs that did not receive public funding (76\% vs $50 \%)$. Similarly, DTPs that received public funding were more likely to offer services free or at no charge (70\% vs 39\%) than DTPs that did not get public funding $(p<0.05)$. And DTPs that received public funding were statistically more likely to offer treatment based on both types of payment options (56\% vs $24 \%$ ) than DTPs that did not receive public funding $(\mathrm{p}<0.05)$.

Table 3B shows differences in payment options offered by DTPs based on public funding allocations and MSA SES characteristics. First, among DTPs located in MSAs with low SES, those that received public funding were statistically more likely to offer treatment services at a fee scale ( 78 vs $47 \%$ ), free of charge (69 vs $37 \%$ ) or both (57 vs $20 \%$ ) than DTPs that did not receive public funding $(\mathrm{p}<0.05)$. And among DTPs located in MSAs with high SES, those that received public funding were statistically more likely to offer treatment services at a fee/pay scale $(65 \%$ vs $40 \%$ ), free of charge (73 vs $38 \%$ ) or both (51 vs $20 \%$ ) than DTPs that did not receive public funding. However, among DTPs that received public funding the differences in payment options offered between those DTPs located in low vs high SES was not significant. 
Am. J. of Economics and Business Administration 4 (1): 72-83, 2012

Table 2: DTP, MSA and state characteristics

\begin{tabular}{|c|c|c|c|c|}
\hline \multicolumn{2}{|c|}{ Level 1: Drug Treatment Provider (DTP) } & \multicolumn{3}{|c|}{$\mathrm{N}=6062$} \\
\hline Variable & Name & Mean & Std Div & Range \\
\hline SCALE & Treatment services are provided based on a pay fee scale. & 0.62 & 0.47 & $0-1$ \\
\hline FREE & Treatment services are provided at no charge (free). & 0.61 & 0.49 & $0-1$ \\
\hline BOTH & Treatment services are provided based on a pay fee scale or at no charge. & 0.47 & 0.50 & $0-1$ \\
\hline PUBFUND & Receives public funds from Federal, State or Local Governments & 0.72 & 0.45 & $0-1$ \\
\hline SADTP & DTP has a substance abuse treatment focus & 0.64 & 0.47 & $0-1$ \\
\hline PCTAD & Percentage of clients diagnosed with an alcohol and drug dependence disorder & 0.56 & 0.29 & $0-1$ \\
\hline SPESER & Mean number of specialized treatment services offered. & 0.24 & 0.21 & $0-1$ \\
\hline ANXSER & Mean number of ancillary treatment services offered. & 0.55 & 0.24 & $0-1$ \\
\hline RESDTP & DTP also offers residential treatment services & 0.36 & 0.48 & $0-1$ \\
\hline DTPSIZE & $\begin{array}{l}\text { Number of clients DTP treats }(1=1-24 ; 2=25-74,3 \\
=75-149,4=150-299,5=300 \text { and more })\end{array}$ & 2.56 & 1.30 & $1-5$ \\
\hline \multicolumn{2}{|c|}{ Level 2: Metropolitan statistical area } & \multicolumn{3}{|l|}{$\mathrm{N}=359$} \\
\hline MSASES & Mean MSA Social Economics Status Category $(1=$ Low, $2=$ Medium, $3=$ High $)$ & 1.95 & 0.60 & $1-3$ \\
\hline \multicolumn{2}{|c|}{ Level 3: State government } & $\mathrm{N}=51$ & & \\
\hline STGOV & Governor is republican (Proxy for State preferences/tastes/altruism) & 0.46 & 0.50 & $0-1$ \\
\hline
\end{tabular}

Table 3A: Treatment affordability by public funding

\begin{tabular}{lllllll}
\hline Public & Mean & Std & Mean & Std & Mean & Std \\
Funding & Fee Scale & Div & Free & Div & Both & Div \\
\hline Yes (1) & 0.76 & 0.43 & 0.70 & 0.46 & 0.56 & 0.50 \\
No (0) & 0.50 & 0.50 & 0.39 & 0.49 & 0.24 & 0.43 \\
\hline
\end{tabular}

Table 3B: Treatment affordability by public funding and by MSA SES

\begin{tabular}{llllllll}
\hline MSA & Public & Mean & Std & Mean & Std & Mean & Std \\
SES & funding & Scale & Div & Free & Div & Both & Div \\
\hline Low (1) & & 0.78 & 0.42 & 0.69 & 0.46 & 0.57 & 0.50 \\
Medium (2) & Yes (1) & 0.78 & 0.42 & 0.69 & 0.46 & 0.56 & 0.50 \\
High (3) & & 0.65 & 0.48 & 0.73 & 0.44 & 0.51 & 0.50 \\
Low (1) & & 0.47 & 0.50 & 0.37 & 0.48 & 0.20 & 0.40 \\
Medium (2) & No (0) & 0.52 & 0.50 & 0.40 & 0.50 & 0.27 & 0.44 \\
High (3) & & 0.40 & 0.49 & 0.38 & 0.49 & & 0.20 \\
\hline
\end{tabular}

Table 3C: Treatment affordability by MSA SES and by state government

\begin{tabular}{|c|c|c|c|c|c|c|c|c|}
\hline $\begin{array}{l}\text { STATE } \\
\text {. }\end{array}$ & MSA & Public & Mean & Std & Mean & Std & Mean & Std \\
\hline GOV & SES & funding & Scale & Div & Free & Div & Both & Div \\
\hline \multirow{7}{*}{ REP (1) } & Low (1) & \multirow{3}{*}{ Yes (1) } & 0.81 & 0.39 & 0.71 & 0.46 & 0.61 & 0.49 \\
\hline & Medium (2) & & 0.76 & 0.43 & 0.69 & 0.46 & 0.57 & 0.50 \\
\hline & High (3) & & 0.74 & 0.44 & 0.48 & 0.50 & 0.38 & 0.50 \\
\hline & Low (1) & \multirow{3}{*}{ No (0) } & 0.50 & 0.50 & 0.36 & 0.48 & 0.21 & 0.41 \\
\hline & Medium (2) & & 0.44 & 0.50 & 0.39 & 0.49 & 0.24 & 0.43 \\
\hline & High (3) & & 0.13 & 0.34 & 0.28 & 0.46 & 0.03 & 0.18 \\
\hline & Low (1) & \multirow{3}{*}{ Yes (1) } & 0.72 & 0.45 & 0.67 & 0.47 & 0.52 & 0.50 \\
\hline \multirow{5}{*}{ DEM (0) } & Medium (2) & & 0.79 & 0.41 & 0.69 & 0.46 & 0.56 & 0.50 \\
\hline & High (3) & & 0.64 & 0.48 & 0.76 & 0.43 & 0.53 & 0.50 \\
\hline & Low (1) & \multirow{3}{*}{ No (0) } & 0.42 & 0.50 & 0.38 & 0.49 & 0.19 & 0.39 \\
\hline & Medium (2) & & 0.58 & 0.49 & 0.41 & 0.49 & 0.29 & 0.46 \\
\hline & High (3) & & 0.44 & 0.50 & 0.40 & 0.49 & 0.22 & 0.41 \\
\hline
\end{tabular}

For example, among these DTPs, offering treatment based on pay/fee scale was 78 vs $65 \%(\mathrm{p}>0.05)$ and offering treatment free was 69 vs $73 \%$ among these DTPs. These results suggest that there are no significant differences $(p>0.05)$ in the returns of public funding made to DTPs in low SES compared to public funding made to DTPs located in MSAs with high SES. Thus there is horizontal equity in public funding.

Table 3C show differences in treatment payment options offered by DTPs based on public funding allocations, SES and State government. The data show, that among DTPs located in States with Republican governors and in MSAs with low SES, those that receive public funding were more likely to offer treatment services at reduced prices than DTPs that did not receive public funding ( 81 vs $50 \% ; 71$ vs $36 \% ; 61$ vs $21 \%$ ). Differences between funds DTPs located in MSAs with low SES in States with Republican governors and those located in States with Democratic governors were not significant (81 vs $72 \%$; 71 vs 
$67 \%$; 61 vs $52 \%$ ). However the differences between funds DTPs located in MSAs with high SES in States with Republican governors and those located in States with Democratic governors were significant ( 74 vs $64 \%$; 48 vs $76 \%$; 38 vs $53 \%$ ). Thus, differences in SES and State governments appear to moderate the treatment prices offered by DTPs that receive funding.

Table 4 shows correlations among the variables of interest. There were high correlations among the level 1 variables examined. In particular, the data show that public funding was positively related to DTPs with high percentage of clients with alcohol and drug treatment; DTPs offering specialized and ancillary services; and negatively related to DTPs located in MSAs with high SES. DTPs located in States that had a Republican governor were less likely to be substance abuse focused, have a high percentage of clients with alcohol and drug dependence, offer specialized and ancillary services and were less likely to offer residential services. They were also less likely to be larger and be located in MSAs with high SES. Since the level of correlation among these variables was relatively high and the model failed to converge within 50,000 iterations, only DTPSIZE was used as a controlling variable at Level 1.

Table 5 presents the results of the regression analyses. First, the second column of the Table shows the logistic regression results for each of the three dependent variables examined. The logistic results show that that public funding and SCALE, FREE and BOTH were significantly $(\mathrm{p}<0.05)$ and positively related. Based on these results, there appears to be little evidence of a flypaper effect. That is, these results show that an increase in public funds for substance abuse treatment will increase the probability that DTPs will offer scale-based pricing, free services, or both by 25 , 22 and $21 \%$, respectively.

The positive and significant relationship between public funding and lower treatment prices offered by DTPs was less dramatic when using HLM, however. Columns 3-7 of Table 5 show the fixed effect results of the five HLM models estimated. First, the results of the unconditional HLM model (HLM1) show that the overall mean price for treatment services $\left(\gamma_{000}\right)$ was significantly significant $(\mathrm{p}<0.05)$ for each of the three prices examined (SCALE, FREE and BOTH). This implies that there were significant mean differences in price behaviors among DTPs. In particular, DTPs were on average $35 \%$ more likely to offer fee/pay scale based prices and about $40 \%$ more likely to offer free services, but they were $54 \%$ less likely to offer both.

The results of the HLM2 shows the treatment prices offered when public funding was included as an independent variable in Level 1. In particular, the coefficient on the grand mean $\left(\gamma_{000}\right)$ shows that DTPs were on average less likely to offer services based on a fee scale $(58 \%)$, free $(61 \%)$, or both $(78 \%)$. These results are consistent with the results of the logistic regression. When looking at the effect of public funding $\left(\gamma_{100}\right)$ on prices offered, the results are also consistent with the results of the logistic regression. In particular, the estimated coefficient of public funding $\left(\gamma_{100}\right)$ shows that DTPs that received public funding were $20 \%$ more likely to offer pay/fee scale-based services, $24 \%$ more likely to offer free services and $20 \%$ more likely to offer both than DTPs that did not receive public funding $(\mathrm{p}<0.05)$.

The results of HLM3, which includes DTP size as controlling variable in level 1 , show that public funding has effects similar to those found in HLM2. That is, DTPs that receive public funding were about $20 \%$ more likely to offer lower treatment prices than DTPs that did not receive public funding. In addition, the results of the HLM3 model show the estimated coefficient on DTPSIZE $\left(\gamma_{200}\right)$ was positive and significant $(\mathrm{p}<0.05)$, indicating that larger DTPs are, on average, $50 \%$ more likely to offer lower treatment prices than smaller DTPs $(\mathrm{p}<0.05)$. These findings suggest that there are economies of scale in the production of affordable treatment services. Thus donors of public funds will get a greater return on their donation if public funds are given to larger DTPs rather than two smaller DTPs. Therefore vertical equity should be considered in public funding allocations.

\begin{tabular}{|c|c|c|c|c|c|c|c|c|c|}
\hline & 1 & 2 & 3 & 4 & 5 & 6 & 7 & 8 & 9 \\
\hline$\overline{\text { FUND }}$ & 1.00 & & & & & & & & \\
\hline SADTP & $0.11^{* *}$ & 1.00 & & & & & & & \\
\hline PCTAD & $0.04 * *$ & 0.23 & 1.00 & & & & & & \\
\hline SPESER & $0.09 * *$ & 0.00 & $0.05 * *$ & 1.00 & & & & & \\
\hline ANXSER & $0.19 * *$ & 0.02 & $0.07 * *$ & $0.29 * *$ & 1.00 & & & & \\
\hline RESDTP & $0.12 * *$ & $0.22 * *$ & $0.19 * *$ & $0.08 * *$ & $0.27 * *$ & 1.00 & & & \\
\hline DTPSIZE & $0.07 * *$ & 0.01 & $-0.19 * *$ & $0.16^{* *}$ & $0.04 * *$ & $-0.33^{* *}$ & 1.00 & & \\
\hline MSASES & -0.03 & 0.00 & -0.02 & $0.06^{* *}$ & $0.06^{* *}$ & -0.01 & 0.01 & 1.00 & \\
\hline STGOV & 0.00 & $-0.13 * *$ & $-0.04 * *$ & $-0.08 * *$ & $-0.08 * *$ & -0.01 & $-0.03 * *$ & $-0.27 * *$ & 1.00 \\
\hline
\end{tabular}


Am. J. of Economics and Business Administration 4 (1): 72-83, 2012

Table 5: Logistic and HLM population-average Odds Ratios (OR) regression results by model

\begin{tabular}{|c|c|c|c|c|c|c|}
\hline \multirow[b]{2}{*}{ Variable } & \multicolumn{6}{|c|}{ Dependent Variable Fee/Pay Scale (Model A) } \\
\hline & $\begin{array}{l}\text { Logistic } \\
\text { OR }\end{array}$ & $\begin{array}{l}\text { HLM1A } \\
\text { OR }\end{array}$ & $\begin{array}{l}\text { HLM2A } \\
\text { OR }\end{array}$ & $\begin{array}{l}\text { HLM3A } \\
\text { OR }\end{array}$ & $\begin{array}{l}\text { HLM4A } \\
\text { OR }\end{array}$ & $\begin{array}{l}\text { HLM5A } \\
\text { OR }\end{array}$ \\
\hline Intercept $\left(\gamma_{000}\right)$ & 0.84 & $1.89^{* *}$ & $0.73 * *$ & $0.43 * *$ & 0.60 & 0.16 \\
\hline $\operatorname{PUBFUND}\left(\gamma_{100}\right)$ & $3.16^{* *}$ & & $3.93 * *$ & $3.75 * *$ & $3.83 * *$ & $11.86^{* *}$ \\
\hline DTPSIZE $\left(\gamma_{200}\right)$ & $1.35^{* *}$ & & & $1.26^{* *}$ & 1.14 & 1.30 \\
\hline $\operatorname{MSASES}\left(\gamma_{010}\right)$ & $0.90^{* *}$ & & & & 0.92 & $1.17 * *$ \\
\hline $\operatorname{STGOV}\left(\gamma_{001}\right)$ & 1.00 & & & & & 12.59 \\
\hline PUBFUND x MSASES $\left(\gamma_{110}\right)$ & & & & & 1.00 & 0.78 \\
\hline DTPSIZE x MSASES $\left(\gamma_{210}\right)$ & & & & & 1.02 & 1.01 \\
\hline PUBFUND x STGOV $\left(\gamma_{101}\right)$ & & & & & & 0.08 \\
\hline DTPSIZE x STGOV $\left(\gamma_{201}\right)$ & & & & & & 0.89 \\
\hline $\operatorname{MSASES} \times \operatorname{STGOV}\left(\gamma_{011}\right)$ & & & & & & 0.63 \\
\hline PUBFUND x MSASES x STGOV $\left(\gamma_{111}\right)$ & & & & & & $1.72 * *$ \\
\hline DTPSIZE x MSASES x STGOV $\left(\gamma_{211}\right)$ & & & & & & 1.00 \\
\hline \multicolumn{7}{|c|}{ Dependent Variable No Charge/Free (Model B) } \\
\hline Intercept $\left(\gamma_{000}\right)$ & 0.71 & $1.52 * *$ & $0.64 * *$ & $0.62 * *$ & 0.64 & 0.99 \\
\hline PUBFUND $\left(\gamma_{100}\right)$ & $3.54 * *$ & & $3.25 * *$ & $3.30 * *$ & 3.45 & 1.95 \\
\hline DTPSIZE $\left(\gamma_{200}\right)$ & 0.97 & & & 1.01 & 1.08 & 1.01 \\
\hline $\operatorname{MSASES}\left(\gamma_{010}\right)$ & 1.00 & & & & 0.99 & 0.91 \\
\hline $\operatorname{STGOV}\left(\gamma_{001}\right)$ & 0.93 & & & & & 0.38 \\
\hline PUBFUND $x$ MSASES $\left(\gamma_{110}\right)$ & & & & & 0.99 & 1.11 \\
\hline DTPSIZE x MSASES $\left(\gamma_{210}\right)$ & & & & & 0.99 & 1.00 \\
\hline PUBFUND x STGOV $\left(\gamma_{101}\right)$ & & & & & & 3.08 \\
\hline DTPSIZE x STGOV $\left(\gamma_{201}\right)$ & & & & & & 1.18 \\
\hline $\operatorname{MSASES} \times \operatorname{STGOV}\left(\gamma_{011}\right)$ & & & & & & 1.21 \\
\hline PUBFUND x MSASES x STGOV $\left(\gamma_{111}\right)$ & & & & & & 0.80 \\
\hline DTPSIZE x MSASES x STGOV $\left(\gamma_{211}\right)$ & & & & & & 0.96 \\
\hline \multicolumn{7}{|l|}{ Dependent Variable Fee/Pay Scale and No } \\
\hline \multicolumn{7}{|l|}{ Charge/Free (Model C) } \\
\hline Intercept $\left(\gamma_{000}\right)$ & $0.35^{* *}$ & 0.84 & $0.29 * *$ & $0.23 * *$ & $0.18^{*}$ & 0.07 \\
\hline PUBFUND $\left(\gamma_{100}\right)$ & $3.85^{* *}$ & & $4.01 * *$ & $3.92 * *$ & $8.84 * *$ & $10.64 * *$ \\
\hline DTPSIZE $\left(\gamma_{200}\right)$ & $1.11^{* *}$ & & & $1.12 * *$ & 0.96 & 1.23 \\
\hline $\operatorname{MSASES}\left(\gamma_{010}\right)$ & 0.94 & & & & 1.04 & 1.23 \\
\hline $\operatorname{STGOV}\left(\gamma_{001}\right)$ & 1.00 & & & & & 5.67 \\
\hline PUBFUND x MSASES $\left(\gamma_{110}\right)$ & & & & & 0.85 & 0.82 \\
\hline DTPSIZE x MSASES $\left(\gamma_{210}\right)$ & & & & & 1.03 & 0.99 \\
\hline PUBFUND x STGOV $\left(\gamma_{101}\right)$ & & & & & & 0.70 \\
\hline DTPSIZE x STGOV $\left(\gamma_{201}\right)$ & & & & & & 0.64 \\
\hline MSASES x STGOV $\left(\gamma_{011}\right)$ & & & & & & 0.73 \\
\hline PUBFUND x MSASES x STGOV $\left(\gamma_{111}\right)$ & & & & & & 1.07 \\
\hline DTPSIZE x MSASES x STGOV $\left(\gamma_{211}\right)$ & & & & & & 1.08 \\
\hline
\end{tabular}

Note: $* \mathrm{p}<0.05, * * \mathrm{p}<0.01$

Next, the results of HLM4, which controls for MSA SES in level 2, show that DTPs that receive public funding were $21 \%$ statistically more likely to offer fee scale services $(\mathrm{p}<0.05)$, but public funding had no effect on offering services for free $(p>0.05)$ and it only had a $10 \%$ effect of offering both price options compared to DTPs that did not receive public funding $(\mathrm{p}<0.05)$. No other variables were found to be statistically significant under HLM4. Finally, when State political preferences were included under HLM5, the results show that public funding had an even less of an effect on DTPs price behaviors. In particular, DTPs that receive public funding were only $8 \%$ more likely to offer treatment services based on either a scale/fee or both (scale and free) than DTPs that did not get public funding $(p<0.05)$. And there was no statistically significant effect of public funding of offering treatment services for free ( $p>0.05)$. Thus, the results of HLM5 show that an increase in public funding appears to increase affordability of treatment services only by about $8 \%$ under HLM models rather than by $25 \%$ under linear models. Furthermore, the results of HLM5 suggest that there is no statistically significant effect of public funding on providing treatment services for free. This suggests the possibility of a flypaper effect on free treatment and/or DTPs deciding to offer more treatment based on a fee based rather than less treatment at no charge. 
Am. J. of Economics and Business Administration 4 (1): 72-83, 2012

Table 6: HLM random effects results

\begin{tabular}{|c|c|c|c|c|c|}
\hline Random effects & HLM 1A & HLM2A & HLM3A & HLM4A & HLM5A \\
\hline \multicolumn{6}{|l|}{ Level 1 and 2} \\
\hline TOTAL $\left(\mathrm{r}_{0}\right)$ & $0.19 * *$ & $0.24 *$ & $0.62 *$ & $0.60 * *$ & $0.57 * *$ \\
\hline PUBFUND $\left(\mathrm{r}_{1}\right)$ & & 0.09 & 0.07 & 0.04 & 0.03 \\
\hline DTPSIZE $\left(\mathrm{r}_{2}\right)$ & & & 0.04 & 0.03 & $0.03 *$ \\
\hline \multicolumn{6}{|l|}{ Level 3} \\
\hline $\operatorname{TOTAL}\left(\mathrm{u}_{00}\right)$ & $0.34 * *$ & $0.25 * *$ & 0.10 & 3.92 & 3.77 \\
\hline PUBFUND $\left(\mathrm{u}_{10}\right)$ & & $0.54 * *$ & $0.62 * *$ & 4.38 & $4.78^{*}$ \\
\hline DTPSIZE $\left(\mathrm{u}_{20}\right)$ & & & 0.01 & 0.33 & 0.29 \\
\hline $\operatorname{MSASES}\left(\mathrm{u}_{01}\right)$ & & & & 0.14 & 0.12 \\
\hline PUBFUND x MSASES $\left(\mathrm{u}_{11}\right)$ & & & & 0.18 & 0.18 \\
\hline DTPSIZE x MSASES $\left(\mathrm{u}_{21}\right)$ & & & & 0.01 & 0.01 \\
\hline \multicolumn{6}{|l|}{ Level 1 and 2} \\
\hline TOTAL $\left(\mathrm{r}_{0}\right)$ & $0.15^{* *}$ & 0.05 & $0.23 *$ & $0.22 * *$ & $0.22 * *$ \\
\hline PUBFUND $\left(\mathrm{r}_{1}\right)$ & & $0.26^{* *}$ & $0.23^{*}$ & $0.12 *$ & $0.12 * *$ \\
\hline DTPSIZE $\left(\mathrm{r}_{2}\right)$ & & & $0.01 *$ & $0.01 *$ & $0.01 *$ \\
\hline \multicolumn{6}{|l|}{ Level 3} \\
\hline $\operatorname{TOTAL}\left(\mathrm{u}_{00}\right)$ & $0.30 * *$ & 0.08 & 0.19 & 4.31 & 4.01 \\
\hline PUBFUND $\left(\mathrm{u}_{10}\right)$ & & $0.15 * *$ & $0.21 *$ & 6.03 & 5.01 \\
\hline DTPSIZE $\left(\mathrm{u}_{20}\right)$ & & & 0.00 & 0.49 & 0.45 \\
\hline $\operatorname{MSASES}\left(\mathrm{u}_{01}\right)$ & & & & 0.16 & 0.15 \\
\hline PUBFUND x MSASES $\left(\mathrm{u}_{11}\right)$ & & & & 0.19 & 0.16 \\
\hline DTPSIZE x MSASES $\left(\mathrm{u}_{21}\right)$ & & & & 0.02 & 0.02 \\
\hline \multicolumn{6}{|l|}{ Level 1 and 2} \\
\hline TOTAL $\left(\mathrm{r}_{0}\right)$ & $0.21 * *$ & 0.20 & $0.50 *$ & $0.47 * *$ & $0.46^{* *}$ \\
\hline PUBFUND $\left(\mathrm{r}_{1}\right)$ & & 0.30 & 0.25 & $0.23^{*}$ & $0.23^{*}$ \\
\hline DTPSIZE $\left(\mathrm{r}_{2}\right)$ & & & 0.02 & 0.03 & $0.03 *$ \\
\hline \multicolumn{6}{|l|}{ Level 3} \\
\hline TOTAL $\left(\mathrm{u}_{00}\right)$ & $0.33^{* *}$ & 0.16 & 0.25 & 10.86 & 10.06 \\
\hline PUBFUND $\left(\mathrm{u}_{10}\right)$ & & 0.13 & 0.19 & 5.39 & 5.34 \\
\hline DTPSIZE $\left(\mathrm{u}_{20}\right)$ & & & 0.00 & 0.87 & 0.78 \\
\hline $\operatorname{MSASES}\left(\mathrm{u}_{01}\right)$ & & & & 0.41 & 0.38 \\
\hline PUBFUND x MSASES $\left(\mathrm{u}_{11}\right)$ & & & & 0.19 & 0.19 \\
\hline DTPSIZE x MSASES $\left(\mathrm{u}_{21}\right)$ & & & & 0.03 & 0.03 \\
\hline
\end{tabular}

$* \mathrm{p}<0.05 ; * * \mathrm{p}<0.01$. Note: Note: Random effects distributed as chi distribution

Table 6 shows the HLM random effects results. The results from HLM1 show that there were statistically significant $(\mathrm{p}<0.05)$ level 1 and 2 overall random effects $\left(\mathrm{r}_{0}\right)$ for all the three dependent variable examined. The results of HLM2 show that there were statistically significant $(\mathrm{p}<0.05)$ public funds random effects at level $3\left(\mathrm{u}_{10}\right)$ for SCALE and FREE, but these effects change under HLM 4 and HLM 5. Thus, the results suggest that there are statistically significant "MSA effects", but no "State effects".

\section{DISCUSSION}

The idea that direct public funding for substance abuse treatment services will benefit society since it will make treatment services more affordable and thus more accessible to people who seek treatment is at the heart of a sound public health policy. But with today's limited government budgets for social programs; governments have decreased their interest in funding public health and social programs and have instead turned to finding more free market solutions to increase affordability of drug treatment services (Hacker, 2009).
The purpose of this study was to examine whether public funding of substance abuse treatment increases its affordability or instead as public economists argue such aid is victim to the flypaper effect and as such will have little effect on the desired outcome.

Although public health policy researchers have enriched the field of behavioral health services by examining the factors that increase the affordability of substance abuse treatment services, limited research has specifically examined the effect that public funding has on the affordability of drug treatment. Economists are quick to caution policy makers that direct public funding might not have the desired effects due to a process they call "the flypaper effect". When this effect occurs, exogenous public aid funding from a higher form of government in a lower system of government, tends to stick where it hits rather than have the desired effect. To the extent that DTPs find ways to redirect the exogenous public aid funding, then such effect will occur and it will not have its intended effect. But if DTPs cannot or choose not to redirect the exogenous aid, then the aid will have its intended effect and there will be less of a flypaper effect. 
The goal of this study is to begin to examine whether there is a "flypaper effect" of public funding for substance abuse treatment services. The results from the logistic regression model shows that there is no evidence of a flypaper effect and that DTPs that receive public funding are more likely to provide treatment services at a lower price, free or both than DTPs that do not receive public funding. However, the results from the HLM models suggest that the effect of public funding on affordability of treatment (i.e., Lower prices) is not as strong as when non-clustering methods are used. In other words, previous research in this area has failed to count for the potential effects that clustered data may have on the results. That is, DTPs are nested within an MSA which are nested within a State. Failing to account for the potential inter correlation of the data violates the linearity assumptions of classical regression models. In other words, simply regressing public funding as an independent variable on the price behaviors of DTPs will violate uncorrelated normality assumptions of the error term. This study begins to make a first step in addressing the aforementioned limitations of previous research in this area by examining the effect of public funds using methods such as HLM which control for clustered level data (Raudenbrush and Brykm, 2002).

The results of the HLM random slope and intercept models do show that public funding increases affordability. However, when controlling factors are included in Level 2, public funding has less of the desired effect on price behavior decisions. Furthermore, when State political preferences are included in Level 3 of the model, public funding has a smaller effect on the desired outcome. In particular, the results show that DTPs that receive public funding are only $8 \%$ more likely to offer lower prices rather than $25 \%$ than DTPs that do not receive public funds.

These results highlight the need for HLM in order to better understand the complex nature of clustered data among DTPs when examining the effects of public funding on the affordability of substance abuse treatment services. Failing to account for clustered data could lead to the Simpson paradox and Ecological fallacies. The results showed that the benefits of public funding vary not only based on DTP characteristics, for example DTP size, but also vary based on the location of the DTP (low SES vs high SES MSA) as well as political environments in the State. Failing to account for these differences, could potentially render limited public funds a useless tool to increase affordability and increase access to substance abuse treatment services.
Limitations and further research: This study examined the effect of public funding on the prices DTPs offer for substance abuse treatment services. This study has several limitations, however. First, this study does answer which type of public funding (State vs Federal, for example) has less of a flypaper effect since these data were not available. If and when such data become available, research should examine these differences. Second, this study centered on the pricing models of private non profits DTPs and did not examine whether and how public DTPs in the area affect the behaviors of these DTPs. For example, it is possible that private non-profit DTPs will offer less free services (i.e., more flypaper effect) in communities where there are a large number of public DTPs. Further research should examine this effect.

Third, the present study only examined one explanatory variable on level 2 and level 3 models. For example, on level 2 MSA socio-demographics could be included. And at level 3, this study only included the Governor political affiliation, but further research could borrow from the rich political science literature to better measure State's preferences for substance abuse treatment. In other words, this study does not capture significant political differences among Republican Governors such as social and fiscal preferences. Forth, the present study does not capture the effect of competition among DTPs within an MSA. For example, four DTPs with $25 \%$ market share each may seen as competitive, but if they are located far from each other, they do not really face competition with each other and thus the effect of public aid will be different. Lastly, it is also possible that consumers are not price elastic to the treatment prices offered by DTPs since clients are often coerced by courts, employers and/or schools to get treatment. So consumers may just want to get treatment from the DTP that is closer to their home or place of work and the price offered by them may be of a second concern.

Although the study does not address all the factors that may determine DTP price behavioral decisions, it is clear from the results, that public funding does have an effect on the affordability of treatment services ranging from $8-25 \%$ and in some cases it has no effect (Model 5B). Therefore, the results of this study also showed that public funding is often a doubled edge sword that should be encouraged only in certain environments and under certain conditions. For example, since aid is fungible DTPs could choose to maximize the number of people receiving treatment at a pay scale rather than maximize the number of people receiving free treatment with the public funding received. In summary, failing to consider the return on 
public funding as measured by lower prices offered by DTPs could potentially be proven an inefficient method of allocating limited funds. Furthermore, it could also have unintended consequences for the people who are most vulnerable and often marginalized in our society and who are in need of affordable substance abuse treatment services.

\section{CONCLUSION}

Our results suggest that the "flypaper effect" previously observed when public funding is transferred from a higher-level government agency to a lower-level government agency may not be generalizable to nonprofit DTPs, which are neither profit-oriented businesses nor government agencies. DTPs that receive public funding are more likely to offer services at no cost or on a sliding scale than DTPs that do not receive public funding. Our results also suggest, however, that failing to account for intercorrelation among clustered individuals or firms operating in the same local or regional environment may result in overestimation of the differences in provision of no-cost or low-cost services between entities receiving public funds and entities not receiving them. The state-level political environment seems to be an especially important influence on the use that DTPs make of public funding. Based on these results, it is recommended that policies aimed at increasing treatment availability retain and perhaps increase the role of public funding of DTPs, but that they focus such funding where it is most likely to result in increased treatment access for the most vulnerable. It is recommended that public policy researchers conduct further studies to determine whether other types of nonprofit publicly funded providers are similarly likely to pass this on to consumers in the form of lower prices for services. Finally, it is recommended that future research on the "flypaper effect" make use of statistical methods that account for clustered or nested data.

\section{REFERENCES}

Bae, S.S. and R.C. Feiock, 2004. The flypaper effect revisited: Intergovernmental grants and local governance. Int. J. Public Admin., 27: 577-596. DOI: $10.1081 / \mathrm{PAD}-120030256$

Brown, J.D., S. Vartivarian and C.E. Alderks, 2011. Child care in outpatient substance abuse treatment facilities for women: Findings from the 2008 National Survey of Substance Abuse Treatment Services. J. Behav. Health Serv. Res., 38: 478-487. PMID: 21293975
Campbell, C.I. and J.A. Alexander, 2006. Availability of services for women in outpatient substance abuse treatment: 1995-2000. J. Behav. Health Serv. Res., 33: 1-19. PMID: 16636905

Campbell, C.I., J.A. Alexander and C.H. Lemak, 2009. Organizational determinants of outpatient substance abuse treatment duration in women. J. Subst. Abuse Treat., 37: 64-72. PMID: 19038526

CDCP, 2007. Best Practices for Comprehensive Tobacco Control Programs. 1st Edn., Department of Health and Human Services, USA., pp: 119.

Cochran B.N., K.M. Peavy and J.S. Robohm, 2007. Do specialized services exist for lgbt individuals seeking treatment for substance misuse? A study of available treatment programs. Subst. Use Misuse, 42: 161-176. PMID: 17366131

Cowell, A., D. McCarty and A. Woodward, 2003. Impact of federal substance abuse block grants on state substance abuse spending: Literature and data review. J. Mental Health Policy Econ., 6: 173-179. PMID: 14713724

Daly, M.C., G.J. Duncan, P. McDonough and D.R. Williams, 2002. Optimal indicators of socioeconomic status for health research. Am. J. Public Health, 92: 1151-1157. PMID: 12084700

Grella, C.E. and L. Greenwell, 2004. Substance abuse treatment for women: Changes in the settings where women received treatment and types of services provided, 1987-1998. J. Behav. Health Serv. Res., 31: 367-383. PMID: 15602139

Hacker, D., 2009. Writer's Reference With Integrated Exercises. 6th Edn., Bedford/st Martins, ISBN-10: 0312608098, pp: 573.

Harwood, H.J. and E. Bouchery, 2004. The Economic Costs of Drug Abuse in the United States, 19922002. 1st Edn., Executive Office of the President, Washington, DC.

Heinrich, C.J. and E. Fournier, 2004. Dimensions of publicness and performance in substance abuse treatment organizations. J. Policy Anal. Manage., 23: 49-70. PMID: 14976993

Hines, J.R. Jr. and R.H. Thaler, 1995. Anomalies: The flypaper effect. J. Econ. Perspectives, 9: 217-226.

Islam, M. and J. Hoddinott, 2009. Evidence of intrahousehold flypaper effects from a nutrition intervention in rural Guatemala. Econ. Dev. Cultural Change, 57: 215-238.

Jacoby, H.G., 2002. Is there an intrahousehold 'Flypaper Effect'? Evidence from a school feeding program. Econ. J., 112: 196-221. 
Karnik, A. and M. Lalvani, 2008. Flypaper effect incorporating spatial interdependence. Rev. Urban Regional Dev. Stud., 20: 86-102. DOI: 10.1111/j.1467-940X.2008.00143.x

Knight, B., 2002. Endogenous federal grants and crowd-out of state government spending: Theory and evidence from the federal highway aid program. Am. Econ. Rev., 92: 71-92.

McLellan, A.T., L. Luborsky, C.P. O’Bried, G.E. Woody and K.A. Druley, 1982. Is treatment for substance abuse effective? J. Am. Med. Associ., 247: 1423-1428. DOI: 10.1001/Jama.1982.03320350027022

Meara, E. and R.G. Frank, 2005. Spending on substance abuse treatment: How much is enough? Addiction, 100: 1240-1248. PMID: 16128713

Moffitt, R.A., 1984. The effects of grants-in-aid on state and local expenditures: The case of AFDC. J. Public Econ., 23: 279-305. DOI: 10.1016/00472727(84)90032-X

Mojtabai, R., 2004. Which substance abuse treatment facilities offer dual diagnosis programs? Am. J. Drug Alcohol Abuse, 30: 525-536. PMID: 15540491

Montoya, I.D., 2006. Differences in drug treatment services based on profit status. J. Psychoactive Drugs, 38: 219-228. PMID: 17165364

Nahra, T.A., J. Alexander and H. Pollack, 2009. Influence of ownership on access in outpatient substance abuse treatment. J. Subst. Abuse Treat., 36: 355-365. PMID: 19339142

Nesbit, T.M. and S.F. Kreft, 2009. Federal grants, earmarked revenues and budget crowd-out: State Highway funding. Public Budget. Finan., 29: 94110. DOI: $10.1111 / \mathrm{j} .1540-5850.2009 .00930 . \mathrm{x}$

O'Brien, C.P. and J. Jaffe, 1992. Addictive States. 1st Edn., Raven Press, New York, ISBN-10: 0881678554, pp: 291.

Olmstead, T. and J.L. Sindelar, 2004. To what extent are key services offered in treatment programs for special populations? J. Subst. Abuse Treat., 27: 9-15. PMID: 15223088
Olmstead, T.A. and J.L. Sindelar, 2005. Does the impact of managed care on substance abuse treatment services vary by provider profit status? Health Serv. Res., 40: 1862-1882. PMID: 16336553

Raudenbush, S.W. and A.S. Bryk, 2002. Hierarchical Linear Models: Applications and Data Analysis Methods. 2nd Edn., Sage Publications, Thousand Oaks, CA., ISBN-10: 076191904X, pp: 485.

Rehm, J., C. Mathers, S. Popova, M. Thavorncharoensap and Y. Teerawattananon et al., 2009. Global burden of disease and injury and economic cost attributable to alcohol use and alcohol-use disorders. Lancet, 373: 22232233. DOI: 10.1016/S0140-6736(09)60746-7

Roemer, J.E. and J. Silvestre, 2002. The "Flypaper Effect" is not an anomaly. J. Public Econ. Theory, 4: 1-17. DOI: 10.1111/1467-9779.00085

Schwallie, D.P., 1989. The Impact of Intergovernmental Grants on the Aggregate Public Sector. 1st Edn., Quorum Books, New York, pp: 153.

Strumpf, K.S., 1996. A Predictive Index for the Flypaper Effect. University of North Carolina.

Tamura, F., 2005. Spending substitution or additional funding? The estimation of endogenous foreign aid fungibility. Brown University.

Turnbull, G.K., 1998. The overspending and flypaper effects of fiscal illusion: Theory and empirical evidence. J. Urban Econ., 44: 1-26. DOI: 10.1006/juec.1997.2056

Walle, D.V.D. and R. Mu, 2007. Fungibility and the flypaper effect of project aid: Micro-evidence for Vietnam. J. Dev. Econ., 84: 667-685. DOI: 10.1016/j.jdeveco.2006.12.005 